Reprod. Nutr. Dévelop., 1987, 27 (5), 955-966.

\title{
Comparaison de deux techniques d'estimation (ninhydrine vs TNBS) de l'azote des acides aminés circulants, appliquées à l'étude de l'absorption intestinale de solutions d'acides aminés libres ou de petits peptides
}

\author{
A. RÉRAT, C. SIMOES NUNES, P. VAISSADE, L. ROGER $\left(^{*}\right)$ \\ Laboratoire de Physiologie de la Nutrition, C.R.J. \\ I.N.R.A., 78350 Jouy-en-Josas, France \\ (*) Laboratoires SOPHARGA, 5, rue Bellini, \\ 92806 Puteaux, France.
}

Summary. A comparison of two techniques (ninhydrine vs TNBS) for estimating circulating amino acid nitrogen : application to the study of absorption of solutions of free amino acids or small peptides in the intestine.

The intestinal absorption of nitrogen from amino acids present in a solution of small peptides or of free amino acids with the same pattern, perfused intraduodenally, has been studied using two analytical techniques $[2,4,6$ trinitrobenzene-1-sulfonic acid (TNBS) after dialysis of the blood vs ninhydrine after chromatography] to determine post-perfusion porto arterial differences and to measure blood flow rate in the portal vein. The results obtained on blood nitrogen level with the chromatographic method were always higher than those obtained using the TNBS method. The differences in the values varied from 8 to $24 \%$ according to post-perfusion time and to blood sample origin (arterial or portal). On the contrary, the absorbed amounts of nitrogen measured by either analytical technique were not very much different : no significant and systematic deviation was found between the two. The TNBS method is thus useful as a preliminary approach to very elaborate studies on intestinal absorption. In these conditions, the nitrogen of amino acids from duodenallyperfused small peptides was absorbed earlier and more rapidly than that from a perfusion of a solution of free amino acids. The amount of nitrogen appearing in the portal vein five hours after perfusion exceeded the perfused amount owing to considerable recycling of endogenous nitrogen.

La composition en acides aminés (aa) du sang est fonction du régime alimentaire ingéré (Pion et al., 1963). Leur teneur dans le sang porte, sang efférent du tractus gastro-intestinal, est généralement plus élevée au cours de la période postprandiale que celle du sang artériel, afférent au tractus gastro-intestinal, et leurs rapports molaires divergent entre le sang porte et le sang artériel (Rérat, 1982), ce qui s'explique par l'absorption intestinale différentielle des divers aa.

Une méthode permettant de décrire la cinétique d'absorption des aa a été mise au point (Rérat et al., 1980). Elle est fondée sur la quantification des diffé- 
rences portoartérielles, par mesure simultanée des concentrations d'aa dans le sang porte et le sang artériel associée à la détermination du débit de sang dans la veine porte.

L'analyse chromatographique liquide des aa est généralement employée, utilisant comme réactif la ninhydrine (Moore et al., 1958). Ce dosage permet de déterminer la concentration molaire de chaque acide aminé, et par là même de calculer la concentration sanguine d'azote provenant des aa, à partir de leur composition moléculaire. Cependant, la préparation des échantillons est très longue. En outre, les volumes d'échantillons nécessaires sont tels qu'ils deviennent limitants du nombre des prises journalières, très élevés lorsqu'il s'agit d'étudier l'absorption intestinale (10 à 20 prélèvements régulièrement espacés au cours de la période postprandiale), et du nombre d'expériences sur un même animal si on veut conserver leur signification physiologique aux paramètres sanguins. Enfin, en raison du nombre de prélèvements journaliers, de longs délais sont nécessaires pour réaliser les mesures, calculer et interpréter les résultats et orienter les travaux futurs. Dans ces conditions, il est opportun de disposer d'une technique simple, rapide et fiable, permettant d'apprécier les variations globales de l'aminoacidémie, à partir desquelles on peut préciser s'il est intéressant de recourir ultérieurement à une expérimentation mettant en œuvre la lourde technique d'analyse chromatographique des échantillons. II existe, en l'occurrence, une technique de dosage utilisant comme réactif l'acide 2, 4, 6, trinitrobenzène-1-sulfonique (TNBS : Satake et al., 1960), présentant ces caractéristiques de simplicité et de fiabilité, mais qui ne permet que de doser l'azote aminé primaire, paramètre légèrement différent de l'azote total présent dans les acides aminés calculé à partir de leurs teneurs azotées respectives.

Le dessein de la présente expérience a été de comparer la validité de la méthode de dosage de l'azote aminé grâce au TNBS pour prévoir l'aminoacidémie en comparant les résultats qu'elle permet d'obtenir à ceux calculés à partir de l'analyse par chromatographie liquide. Ces deux techniques d'analyse biochimique ont été appliquées à des échantillons sanguins récoltés afin de mesurer l'absorption après perfusion duodénale de solutions de peptides ou d'acides aminés libres de même composition.

\section{Matériel et Méthodes.}

Animaux. - Six porcs mâles castrés de la race Large White ont été utilisés. Pendant un mois avant l'expérience, ils ont reçu deux fois par jour à $9 \mathrm{~h}$ et $17 \mathrm{~h}$, un régime alimentaire équilibré (800-1 $000 \mathrm{~g}$ par repas), dont la composition est fournie dans le tableau 1 . Le gain moyen quotidien des animaux pendant cette période a été de $600 \mathrm{~g}$ à $700 \mathrm{~g} / \mathrm{jour}$. Chaque animal a été muni d'une sonde électromagnétique autour de la veine porte pour mesurer le débit sanguin, et de deux cathéters, I'un placé dans la veine porte (Arsac et Rérat, 1962) et I'autre dans le tronc brachiocéphalique gauche par l'artère carotide (Rérat et al., 1980). Au cours de l'intervention chirurgicale, la préparation a été complétée par l'implantation d'un cathéter permanent dans le duodénum. Un cathéter aseptique en sylastic 
(1,98 $\mathrm{mm}$ de diamètre intérieur) est introduit dans le duodénum grâce à un orifice pratiqué au centre d'une suture en bourse (soie $n^{\circ} 4$ ) préparée à l'avance à $10 \mathrm{~cm}$ en aval du pylore. La longueur introduite dans le duodénum, orientée vers sa partie distale, est de 3 à $4 \mathrm{~cm}$. Les fils de la bourse sont serrés autour du cathéter. Celui-ci est fixé à la paroi séro-musculaire du duodénum en amont de son implantation, à l'aide d'un nœud auto-serreur de façon à être situé parallèlement au duodénum. Une ouverture abdominale est pratiquée pour le cathéter en regard du duodénum et les extrêmités des fils de fixation sont utilisées pour attacher à la paroi abdominale le duodénum au point d'implantation du cathéter. Le reste de l'opération chirurgicale a été exécuté selon Rérat et al. (1980).

TABLEAU 1

Composition du régime alimentaire

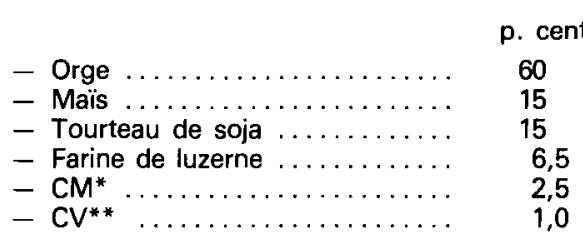

Caractéristiques nutritionnelles :

- Matière sèche :

- Matières azotées totales :

87,8 p. cent

17,0 p. cent M.S.

- Energie brute :

- Matières grasses :

$4,496 \mathrm{Kcal} / \mathrm{kg} \mathrm{M} . \mathrm{S}$.

- Cellulose brute :

6,2 p. cent M.S.

* CM : complément minéral (Henry et Rérat, 1964).

** CV : complément vitaminique (Henry et Rérat, 1964).

Perfusions duodénales. - Avant l'intervention chirurgicale et jusqu'à la fin de l'expérience, les animaux $(57,0 \pm 0,99 \mathrm{~kg}$ poids vif) ont été placés dans des cages permettant un accès facile aux cathéters. Les séries expérimentales ont commencé entre 6 et 8 jours après l'implantation de la sonde et des cathéters et quand les animaux ont recouvré un appétit et un développement normaux.

Chaque animal a été soumis à quatre essais, se succédant à intervalles de 3-4 jours, débutant dans la matinée $(9 \mathrm{~h})$ après un jeûne de $18 \mathrm{~h}$, et se prolongeant pendant $5 \mathrm{~h}$. Chacun de ces essais était caractérisé par la nature du produit perfusé [mélange peptidique (PEP) versus solution d'acides aminés libres de même composition (AAL), tabl. 2] et par la quantité perfusée ( $55 \mathrm{~g}$ dans $500 \mathrm{ml}$ d'eau, $110 \mathrm{~g}$ dans $1000 \mathrm{ml}$ d'eau). Le mélange peptidique (tabl. 2) était formé d'un mélange d'hydrolysats ménagés (Roger et al., 1979 ; Brule et al., 1980a, b) obtenus à $38^{\circ} \mathrm{C}$ par l'action de la chymotrypsine et de la trypsine en réacteur enzymatique à membranes sur les protéines de lactosérum (1/3) et sur la caséine $(1 / 3)$ et d'un mélange de peptides non phosphorylés extrait de l'hydrolysat enzymatique de caséine (1/3). La teneur en vitamines et en oligoéléments des deux 
solutés était la même et celle des minéraux a été ajustée afin d'égaliser leur pression osmotique. La perfusion des solutés expérimentaux a duré $30 \mathrm{mn}$.

La séquence des perfusions a été réalisée selon un double carré Latin pour maîtriser l'influence de la sućcession des traitements expérimentaux.

\section{TABLEAU 2}

Composition en acides aminés (aa) et distribution du poids moléculaire des peptides (P.M.) de l'hydrolysat de protéines laitières.

\begin{tabular}{|c|c|c|c|c|c|c|c|c|c|c|c|}
\hline $\begin{array}{l}\text { Composition } \\
\text { en A.A. (\%) }\end{array}$ & $\begin{array}{l}\text { ILE } \\
\text { TYR } \\
\text { ALA }\end{array}$ & $\begin{array}{l}5,1 ; \\
4,9 ; \\
3,6 ;\end{array}$ & $\begin{array}{l}\text { LEU } \\
\text { THR } \\
\text { ASP }\end{array}$ & $\begin{array}{r}10,1 ; \\
4,4 \\
8,4\end{array}$ & $\begin{array}{l}\text { LYS } \\
\text { TRP } \\
\text { GLU }\end{array}$ & $\begin{array}{r}8,5 ; \\
1,8 ; \\
15,9 ;\end{array}$ & $\begin{array}{l}\text { MET } \\
\text { VAL } \\
\text { GLY }\end{array}$ & $\begin{array}{l}2,3 ; \\
5,9 ; \\
1,8 ;\end{array}$ & $\begin{array}{l}\text { CYS } \\
\text { ARG } \\
\text { PRO }\end{array}$ & $\begin{array}{ll}1,6 ; & \mathrm{PHE} \\
3,3 ; & \mathrm{HIS} \\
8,4 ; & \text { SER }\end{array}$ & $\begin{array}{l}4,6 ; \\
2,5 ; \\
4,7 ;\end{array}$ \\
\hline $\begin{array}{c}\text { Distribution } \\
\text { P.M. } \\
\text { (\%) }\end{array}$ & & P.M. & $3^{1,500}$ & & & $10>P$. & $\frac{M .}{17}<$ & 5,000 & 5,000 & $>\underset{3}{\text { P.M. }}<$ & 6,000 \\
\hline
\end{tabular}

A.A. libres (\% du mélange)

Teneur en azote $(\%): 12,678$.

(1) Plus de $60 \%$ ayant moins de 5 résidus d'aa.

Mesures. - Le jour de chaque perfusion, le débit sanguin portal a été enregistré de façon continue et le sang a été prélevé $(7 \mathrm{ml} /$ voie) simultanément par les cathéters porte et carotide, tous les quarts d'heure durant la 1 re heure, toutes les $30 \mathrm{mn}$ durant les $2^{e}$ et $3^{e}$ heures, et une fois à la $4^{e}$ et la $5^{e}$ heure.

La concentration sanguine d'azote aminé ( $N$ aminé) a été déterminée extemporanément sur un appareil automatique selon la technique à l'acide 2, 4, 6, trinitrobenzène-1-sulfonique après dialyse (TNBS : Palmer et Peters, 1966, 1969). Le sang total est dilué par une solution de $\mathrm{NaCl} 0,9 \%$, puis est soumis à une dialyse qui remplace avantageusement dans une analyse automatisée les différentes opérations de la centrifugation, de la récupération du surnageant et des dilutions dans une analyse manuelle. Seul le liquide de contre dialyse est récupéré. II est constitué par un milieu tamponné borax $(\mathrm{pH}=9,5)$ qui s'est enrichi en petites molécules, dont les acides aminés libres du sang à l'exclusion des plus grosses molécules comme les protéines ou l'hémoglobine. On le place alors en présence de l'acide 2,4,6 trinitrobenzène-1-sulfonique qui réagit avec le groupement $\mathrm{NH}_{2}$ terminal d'un acide aminé pour former un dérivé trinitrophényl de cet acide aminé, de couleur orange, après passage dans un bain-marie $\left(60^{\circ} \mathrm{C}\right)$. L'acidification du milieu réactionnel par addition d'acide chlorhydrique $1 \mathrm{~N}$, rend stable ce composé de couleur jaune. Il est alors analysé dans une cuve de spectrophotomètre à $420 \mathrm{~nm}$ de longueur d'onde, en comparaison avec des solutions standard d'un acide aminé (Rérat et al., 1985) choisi comme référence. II s'agit ici de solutions de L-leucine, par commodité étant donné sa réponse assez peu différente de celle obtenue avec une solution plus onéreuse d'un mélange de 18 acides aminés.

Pour déterminer la teneur en acides aminés individuels, $2 \mathrm{ml}$ de sang par cathéter ont été isolés à chaque prélèvement et mélangés dans un tube à $5,5 \mathrm{ml}$ 
d'une solution (acide sulfosalicylique $2 \mathrm{H}_{2} \mathrm{O}, 64,7 \mathrm{~g} / \mathrm{l}$; thiodiglycol $6 \mathrm{ml} / \mathrm{l}$; norleucine 200 micromoles/l). Ces tubes, dûment agités, sont conservés à $-80^{\circ} \mathrm{C}$. L'extraction des acides aminés libres est réalisée par broyage ultrasonique 3 fois pendant une minute à l'aide de pulsions à mi-temps, broyage suivi d'une centrifugation $(6000 \mathrm{~g} ; 20 \mathrm{~min})$, collecte du surnageant et ajustement à pH 2,2. La détermination des aa a été faite sur un auto-analyseur (Moore, Spackman et Stein, 1958), le réactif utilisé étant la ninhydrine.

Calculs et analyses statistiques. - Les données sont exprimées en moyennes et erreurs standards de la moyenne ( \pm SEM). Le test $t$ de Student est utilisé pour évaluer la signification des variations (Snedecor et Cochran, 1967). L'appréciation de l'apparition nette d'azote aminé et d'acides aminés dans la circulation portale est calculée grâce aux formules (Rérat et al., 1980) :

$$
\begin{gathered}
q=(C p-C a) D d t \\
Q=\Sigma_{t_{0}}^{t_{1}} a
\end{gathered}
$$

où q est la quantité absorbée durant le temps court $\mathrm{dt}(5 \mathrm{~min})$ pendant lequel les paramètres étudiés peuvent être considérés comme constants, $\mathrm{Cp}$ est la concentration portale, Ca la concentration artérielle, $\mathrm{D}$ le débit sanguin portal et $\mathrm{O}$ la quantité apparaissant durant la période de temps écoulée entre $t_{0}$ et $t_{1}$.

Cette mesure ne permet pas d'apprécier l'absorption totale, mais seulement le flux net des nutriments vers la veine porte, puisque certains nutriments provenant de la lumière intestinale ou du sang artériel peuvent être métabolisés dans la paroi intestinale. Pour un nutriment perfusé dans le duodénum, les valeurs mesurées correspondent à l'absorption en excès par rapport au métabolisme de la paroi quand la différence portoartérielle est postitive, ou au métabolisme de la paroi en excès relativement à l'absorption quand la différence portoartérielle est négative. D'autre part, la synthèse par le tissu intestinal d'une substance non perfusée peut être mesurée de cette façon (Rérat et al., 1980).

\section{Résultats.}

1. Débit sanguin portal. - La nature de la perfusion n'a eu aucune influence sur le niveau et l'évolution du débit sanguin : pour l'ensemble des animaux et des expériences $(\mathrm{n}=24)$, le débit portal moyen sur $5 \mathrm{~h}$ s'élève à $2310 \pm 99 \mathrm{ml} / \mathrm{min}$ (soit $34,4 \pm 1,4 \mathrm{ml} / \mathrm{kg} / \mathrm{min}$ ), les variations postperfusion observées allant dans le même sens que celles déjà décrites en situation postprandiale (Rérat et al., 1985).

2. Evolution des concentrations sanguines de l'azote. - L'évolution de la concentration d'azote aminé total, analysé par la technique TNBS, et ses variations selon les modalités de la perfusion sont présentées dans la figure 1.

La perfusion duodénale des solutés expérimentaux, quelles que soient leur nature et leur quantité, se traduit par une élévation rapide et importante des concentrations d' $\mathrm{N}$ aminé dans le sang portal. Celles-ci atteignent un maximum entre 30 et $60 \mathrm{~min}$ après le début de la perfusion. A cette élévation succède une 

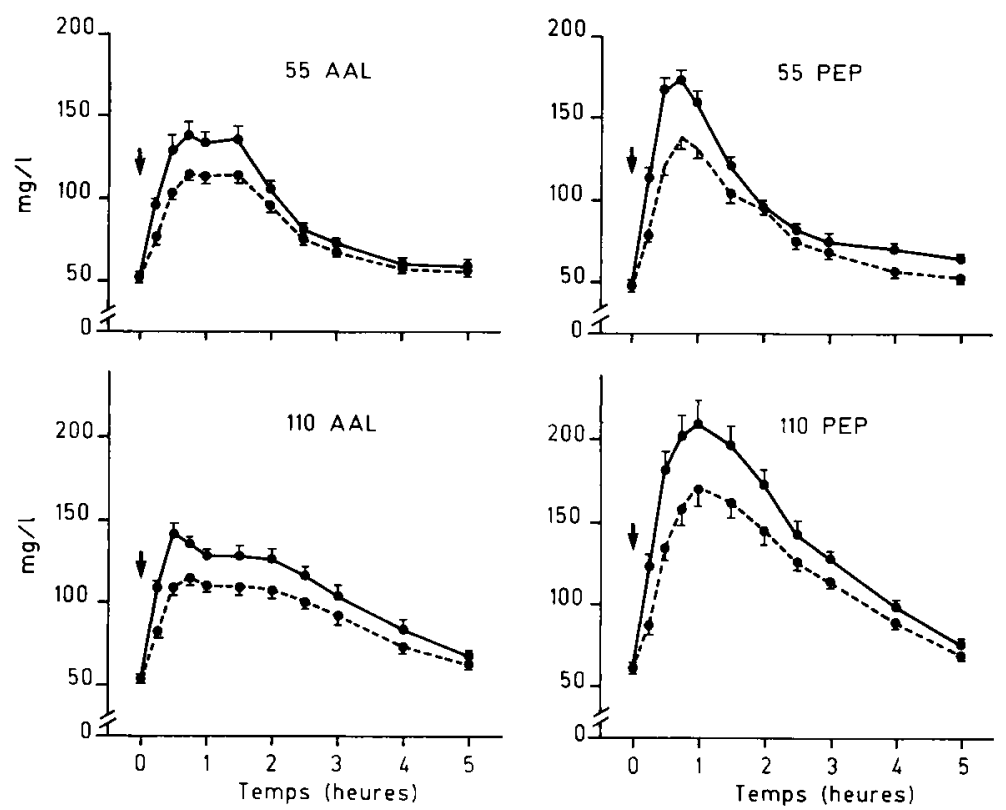

FIG. 1. - Evolution temporelle des concentrations sanguines portales ( et artérielles $\left({ }^{-}-\ldots\right)$ (moyenne \pm écart type de la moyenne en $\left.\mathrm{mg} / \mathrm{l}\right) \mathrm{d}^{\prime}$ azote aminé déterminé selon la méthode TNBS après perfusion duodénale de solutions contenant 55 ou $110 \mathrm{~g}$ de peptides (PEP) ou d'acides aminés libres (AAL) de même composition. (†) Début de la perfusion.

décroissance progressive jusqu'à la cinquième heure. La concentration artérielle présente des variations parallèles, mais atténuées. Le maximum atteint est généralement plus élevé et plus tardif, et les différences portoartérielles sont plus marquées et plus durables quand s'élève la quantité perfusée et quand au lieu d'AAL sont perfusés les PEP. Les différences entre les perfusions de 55 et $110 \mathrm{~g}$ d'AAL sont surtout marquées par la persistance des différences portoartérielles.

Le retour des concentrations portales au niveau des concentrations artérielles, qui signe la fin de la période d'absorption (Rérat, 1981), n'est pas obtenu au cours des cinq heures que dure la période d'observation, les différences portoartérielles rémanentes à la $5^{\mathrm{e}}$ heure variant entre 3,8 et $14,5 \%$ de la concentration initiale moyenne (CIM).

Les principales variations enregistrées pour les teneurs sanguines d'azote des acides aminés (fig. 2), teneurs calculées à partir de l'analyse chromatographique (somme des concentrations de chaque acide aminé multipliées par leurs teneurs individuelles en azote), sont analogues, à l'échelle près, à celles trouvées pour l'azote aminé.

Les valeurs trouvées à l'aide du TNBS sont, selon le temps et la voie de prélèvement, systématiquement inférieures (de 8 à $24 \%$ ) à celles obtenues par chromatographie (tabl. 3). II n'existe pas un écart parallèle au cours du temps pour les valeurs trouvées par les deux méthodes dans le sang portal et le sang artériel. L'écart moyen pour l'ensemble des prélèvements est de $14,3 \%$ dans le sang por- 

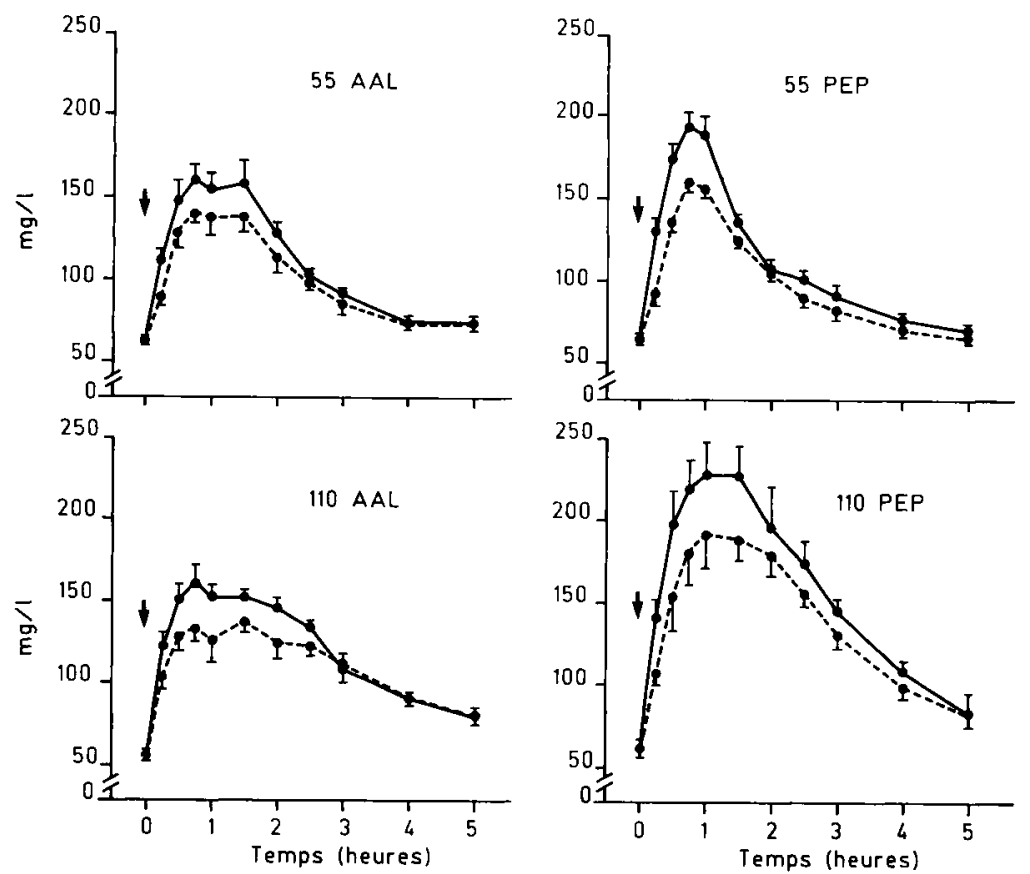

FIG. 2. - Evolution temporelle des concentrations sanguines portales (

) et arterielles

( - - - ) (moyenne \pm écart type de la moyenne en $\mathrm{mg} / \mathrm{l}$ ) de l'azote des acides aminés déterminés par la méthode de chromatographie, après perfusion duodénale de solutions contenant 55 ou $110 \mathrm{~g}$ de peptides ou d'acides aminés libres (AAL) de méme composition. (†) Début de la perfusion.

\section{TABLEAU 3}

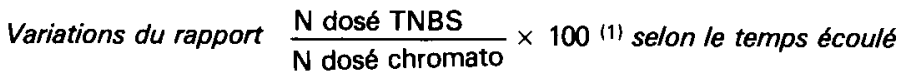

depuis le début de la perfusion et la voie sanguine.

\begin{tabular}{ccccccccccccc}
\hline $\begin{array}{c}\text { Temps écoule } \\
\text { après la }\end{array}$ & & & & & & & & & & & & \\
perfusion (min) & 0 & 15 & 30 & 45 & 60 & 90 & 120 & 150 & 180 & 240 & 300 & $\mathrm{~m}^{(2)}$ \\
\hline VP & 83,2 & 86,9 & 92,2 & 87,5 & 86,6 & 85,5 & 86,5 & 82,7 & 87,5 & 83,3 & 80,9 & 85,69 \\
& $\pm 2,3$ & $\pm 1,0$ & $\pm 1,8$ & $\pm 2,0$ & $\pm 1,8$ & $\pm 1,1$ & $\pm 1,3$ & $\pm 1,8$ & $\pm 3,7$ & $\pm 2,4$ & $\pm 1,7$ & $\pm 0,70$ \\
AC & 78,0 & 83,3 & 85,6 & 85,9 & 85,6 & 83,5 & 87,5 & 81,3 & 82,5 & 79,7 & 76,4 & 82,62 \\
& $\pm 1,1$ & $\pm 1,9$ & $\pm 1,8$ & $\pm 1,4$ & $\pm 1,5$ & $\pm 1,1$ & $\pm 1,9$ & $\pm 1,6$ & $\pm 1,6$ & $\pm 1,2$ & $\pm 1,2$ & $\pm 0,65$ \\
\hline
\end{tabular}

(1) 4 données par voie sanguine (chacune représentant $\frac{\mathrm{N} \text { TNBS moyen }}{\mathrm{N} \text { Chromato. moyen }}$ pour 6 animaux) et par temps de prélèvement (moyenne \pm SEM).

(2) $m$ (sur 44 données \pm SEM). Difference significative au seuil de 5 p. 1,000. 
tal et de $17,4 \%$ dans le sang artériel (différences statistiquement significatives, $P<0,05)$.

3. Evolution des quantités d'azote absorbées. - Les quantités absorbées selon le temps écoulé après la perfusion sont rapportées dans le tableau 4, soit sous forme d'azote aminé (méthode TNBS), soit sous forme d'azote total présent dans les acides aminés du sang (méthode chromatographique).

Malgré les nombreuses divergences d'importance variable enregistrées dans les concentrations selon la méthode utilisée, il apparaît que les valeurs quantitatives trouvées par l'une ou l'autre méthode sont voisines. Les écarts ont tendance à s'accentuer avec le temps écoulé depuis le début de la perfusion : ainsi, à la cinquième heure, quelle que soit la nature du perfusat, les valeurs trouvées par chromatographie sont de 11 à $14 \%$ plus élevées après perfusion de $55 \mathrm{~g}$ que celles trouvées par la méthode TNBS. Elles sont inférieures de 2 à $6 \%$ après perfusion de $110 \mathrm{~g}$. En aucun cas, les écarts entre les deux méthodologies n'atteignent le seuil de signification de $5 \%$.

TABLEAU 4

Evolution des quantités d'azote absorbé sous forme d'acides aminés par tranche de temps selon la nature de la perfusion et de la méthode d'analyse

(TNBS vs chromatographie).

\begin{tabular}{|c|c|c|c|c|c|c|c|}
\hline \multirow{3}{*}{$\begin{array}{c}\text { Quantité } \\
\text { perfusée }\end{array}$} & \multirow{3}{*}{$\begin{array}{c}\text { Mat. sèche }(g) \\
N(g)\end{array}$} & \multicolumn{2}{|c|}{55} & \multicolumn{2}{|c|}{110} & \multirow{2}{*}{\multicolumn{2}{|c|}{$\begin{array}{c}\text { Signification } \\
\text { des différences } \\
\text { entre niveaux } \\
55 \text { et } 110 \mathrm{~g}\end{array}$}} \\
\hline & & \multirow{2}{*}{7,49} & \multirow{2}{*}{$\begin{array}{l}7,38 \\
\text { PEP }\end{array}$} & \multirow{2}{*}{14,97} & \multirow{2}{*}{$\frac{14,76}{\text { PEP }}$} & & \\
\hline & & & & & & AAL & PEP \\
\hline $\begin{array}{l}\text { Période } \\
\text { après } \\
\text { perfusion } \\
\text { (min) }\end{array}$ & $\begin{array}{c}\text { Méthode } \\
\text { de } \\
\text { dosage }\end{array}$ & & & & & & \\
\hline $0-30$ & $\begin{array}{l}\text { TNBS } \\
\text { Chr. }\end{array}$ & $\begin{array}{l}1,29 \pm 0,10^{\mathrm{A}} \\
1,24 \pm 0,18^{\mathrm{A}}\end{array}$ & $\begin{array}{l}2,19 \pm 0,39 \mathrm{~B} \\
2,29 \pm 0,35^{\mathrm{B}}\end{array}$ & $\begin{array}{l}1,67 \pm 0,26^{x} \\
1,39 \pm 0,28^{x}\end{array}$ & $\begin{array}{l}2,22 \pm 0,26^{x} \\
2,47 \pm 0,45^{x}\end{array}$ & $\begin{array}{l}\text { NS } \\
\text { NS }\end{array}$ & $\begin{array}{l}\text { NS } \\
\text { NS }\end{array}$ \\
\hline $30-60$ & $\begin{array}{c}\text { TNBS } \\
\text { Chr. } \\
\text { TNBS }\end{array}$ & $\begin{array}{l}1,61 \pm 0,31^{\mathrm{A}} \\
1,70 \pm 0,37^{\mathrm{A}} \\
2,58+0,31^{\mathrm{A}}\end{array}$ & $\begin{array}{l}2,69 \pm 0,35^{\mathrm{B}} \\
2,59 \pm 0,31^{\mathrm{B}} \\
2,50 \pm 0,22^{\mathrm{A}}\end{array}$ & $\begin{array}{l}1,55 \pm 0,26^{x} \\
2,21 \pm 0,54^{x} \\
2,42 \pm 0,37^{x}\end{array}$ & $\begin{array}{l}3,78 \pm 0,39 y \\
3,10 \pm 0,37 \times \\
4,95 \pm 0,48 y\end{array}$ & $\begin{array}{l}\text { NS } \\
\text { NS } \\
\text { NS }\end{array}$ & $\begin{array}{l}\text { NS } \\
\text { NS } \\
* * *\end{array}$ \\
\hline $60-120$ & $\begin{array}{c}\text { TNBS } \\
\text { Chr. }\end{array}$ & $2,95 \pm 0,73 \mathrm{~A}$ & $2,43 \pm 0,47^{\mathrm{A}}$ & $2,68 \pm 0,37^{x}$ & $5,15 \pm 0,93^{\gamma}$ & NS & $* *$ \\
\hline $120-180$ & $\begin{array}{l}\text { TNBS } \\
\text { Chr. }\end{array}$ & $\begin{array}{l}0,92 \pm 0,11^{\mathrm{A}} \\
1,19 \pm 0,12^{\mathrm{A}}\end{array}$ & $\begin{array}{l}0,94 \pm 0,16^{\mathrm{A}} \\
1,54 \pm 0,55^{\mathrm{A}}\end{array}$ & $\begin{array}{l}2,10 \pm 0,10^{x} \\
1,89 \pm 0,28^{x}\end{array}$ & $\begin{array}{l}2,78 \pm 0,33^{X} \\
2,75 \pm 0,24^{\curlyvee}\end{array}$ & $c^{* * *}$ & $\begin{array}{l}* * * \\
\text { NS }\end{array}$ \\
\hline $180-240$ & TNBS & $0,68 \pm 0,13^{A}$ & $0,72 \pm 0,18^{A}$ & $1,42 \pm 0,24^{x}$ & $1,77 \pm 0,20^{x}$ & 6 * & $* *$ \\
\hline & Chr. & $0,82 \pm 0,18^{A}$ & $1,05 \pm 0,37^{A}$ & $0,81 \pm 0,22^{x}$ & $1,42 \pm 0,11^{Y}$ & NS & ** \\
\hline $240-300$ & TNBS & $0,57 \pm 0,23^{A}$ & $0,40 \pm 0,10^{\mathrm{A}}$ & $0,97 \pm 0,21 x$ & $1,04 \pm 0,21^{x}$ & NS & NS \\
\hline $0-300$ & TNBS & $\begin{array}{l}0,61 \pm 0,18^{A} \\
7,65 \pm 0,58^{A}\end{array}$ & $\begin{array}{l}0,80 \pm 0,20^{A} \\
9,43 \pm 1,18^{A}\end{array}$ & $\begin{array}{r}0,53 \pm 0,10 x \\
10,14 \pm 1,77^{x}\end{array}$ & $16,15 \pm 1,26 y$ & NS & $* *$ \\
\hline & Chr. & $8,52 \pm 1,23^{A}$ & $10,78 \pm 1,58 \mathrm{~A}$ & $9,53 \pm 0,63^{x}$ & $15,84 \pm 1,58 v$ & NS & \\
\hline
\end{tabular}

TNBS : Acide 2, 4, 6 trinitrobenzène-1-sulfonique - Chr. : Chromatographie ;

$A A L$ acides aminés libres ; PEP : petits peptides.

Signification statistique : NS : Non significatif, ${ }^{*} P<0,05$; ${ }^{*} P<0,01 ;{ }^{* * *} P<0,001$.

Pour un même niveau, un même temps, et une même méthode, les valeurs affectées d'une lettre différente sont significativement différentes (majuscules $P<0,05 ;$ minuscules $P<0,01$ ). II n'existe aucune différence significative (au seuil de $5 \%$ ) entre les valeurs obtenues par les deux méthodes. 
La perfusion se traduit par l'apparition précoce de l'azote des acides aminés dans la veine porte puisque, quels que soient le type et le niveau de perfusion et la méthodologie utilisée pour le dosage de I'N, 65 à $69 \%$ des quantités totales absorbées en $5 \mathrm{~h}$ le sont au cours des deux premières heures. C'est particulièrement au cours de cette période que les différences en faveur de la solution de peptides sont marquées : après perfusion de $55 \mathrm{~g}$, ces différences sont significatives dans les intervalles de temps $0-30 \mathrm{~min}$ et $30-60 \mathrm{~min}$ selon les deux méthodes ; après perfusion de $110 \mathrm{~g}$, elles le sont entre 30 et 60 min selon la méthode TNBS, et entre 0 et $60 \mathrm{~min}$, ainsi qu'entre 60 et 120 min selon les deux méthodes. Mais alors que ces différences entre les deux types de solutions restent importantes jusqu'à la $5^{e}$ heure $\left(P<0,05\right.$ aux $3^{e}$ et $4^{e}$ heures selon la méthode chromatographique) dans le cas du niveau le plus élevé, elles s'estompent et disparaissent dans le cas de la dose la plus faible. II en résulte qu'après $5 \mathrm{~h}$, les quantités totales absorbées ne sont pas significativement différentes entre les 2 types de perfusion au niveau de $55 \mathrm{~g}$, mais le sont au niveau de $110 \mathrm{~g}$, quelle que soit la méthodologie utilisée.

L'influence du niveau de perfusion sur les quantités absorbées est très marquée lorsque la solution est composée de PEP, l'absorption étant significativement plus élevée entre la $2^{\mathrm{e}}$ et la $5^{\mathrm{e}}$ heure pour le niveau $110 \mathrm{~g}$ que pour le niveau $55 \mathrm{~g}$ selon la méthode TNBS (et seulement à la $2^{\mathrm{e}}$ et $4^{\mathrm{e}}$ heures selon la méthode chromatographique). Par contre, après perfusion d'AAL, les quantités absorbées sont à peine plus grandes au niveau $110 \mathrm{~g}$ qu'au niveau $55 \mathrm{~g}$, les seules différences significatives étant enregistrées à la $3^{\mathrm{e}}$ heure selon les deux méthodes et à la $4^{e}$ heure selon la méthode TNBS. II apparaît ainsi que l'évolution des quantités absorbées selon le type et la nature de la perfusion est identique, quelle que soit la méthode de dosage utilisée pour apprécier l'azote des acides aminés. Toutefois, les différences liées à ces facteurs n'ont pas toujours le même degré de signification statistique.

Les quantités d'azote provenant des acides aminés absorbés en cinq heures sont généralement supérieures aux quantités perfusées sauf dans le cas de la perfusion de $110 \mathrm{~g}$ d'AAL. Le surplus absorbé, représentant le recyclage d'azote endogène (Rérat et al., 1977), est relativement plus important pour la plus faible dose perfusée. II varie selon la méthode entre $0,16 \mathrm{~g}$ (TNBS) et $1,03 \mathrm{~g}$ (chromatographie) après perfusion de $55 \mathrm{~g} \mathrm{AAL}$, entre $2,05 \mathrm{~g}$ (TNBS) et $3,40 \mathrm{~g}$ (chromatographie) après perfusion de $55 \mathrm{~g}$ PEP, et entre 1,39 $\mathrm{g}$ (TNBS) et $1,08 \mathrm{~g}$ (chromatographie) après perfusion de $110 \mathrm{~g}$ de PEP. Cinq heures après perfusion de $110 \mathrm{~g}$ d'AAL, seulement 64 (chromatographie) à $68 \%$ (TNBS) de l'azote de la solution perfusée est apparu dans la veine porte (contre 107 à $109 \%$ dans le cas de la solution PEP).

\section{Discussion.}

Les principes de la méthode d'étude de l'absorption des nutriments ont déjà été décrits par ailleurs ainsi que ses modalités (Rérat et al., 1980). L'étude de la disparition des nutriments de la lumière digestive, généralement utilisée en gas- 
troentérologie, ne permet pas de préciser la nature des nutriments d'origine alimentaire apparaissant dans le " milieu intérieur ", ni leur cinétique d'apparition puisqu'ils peuvent avoir été métabolisés ou détruits au cours de la digestion et de l'absorption. La méthode ici présentée rend compte de la nature et des quantités de nutriments mis à disposition des tissus après modification ou captation par l'entérocyte (Rérat et al., 1980).

Pour mettre en œuvre cette méthodologie, il faut évidemment disposer de techniques fiables, aussi bien de débitmétrie portale (Rérat et Vaugelade, 1983) que d'analyse sanguine des nutriments étudiés.

En ce qui se rapporte aux acides aminés, la chromatographie liquide sur colonnes échangeuses d'ions permettant de les séparer, de les caractériser et de les quantifier à l'aide d'une réaction colorée à la ninhydrine a largement fait ses preuves. Cette technique peut servir de technique de référence pour définir les concentrations molaires de chaque acide aminé dans le sang et d'en déduire la concentration sanguine d'azote $\alpha$-aminé et total provenant des acides aminés.

Le dosage de l'azote aminé à l'aide du TNBS peut-il constituer une bonne technique de remplacement de l'analyse chromatographique à l'aide de ninhydrine pour évaluer l'aminoacidémie ? Certes, cette technique a le mérite d'être simple et rapide; en outre, le réactif coloré ne réagit pas en présence d'ammoniaque et d'urée (Palmer et Peters, 1966). Le fait que le TNBS réagisse avec d'autres groupements aminés que ceux des aminoacides ne constitue pas un désavantage sérieux pour la mesure de l'aminoacidémie sanguine puisque les amines primaires ne sont trouvées dans le sang qu'à une fréquence faible ou en très petite quantité. Par contre, ce réactif présente une sensibilité variable selon les acides aminés : ainsi cette sensibilité est de $75 \%$ vis-à-vis des aa basiques, de $50 \%$ vis-àvis des aa acides et nulle vis-à-vis des iminoacides (Waring et Bolton, 1967 ; Prenton et London, 1967). II est ainsi évident que les valeurs absolues d'azote aminé trouvées à l'aide de ce réactif ne peuvent strictement coïncider avec celles découlant des valeurs individuelles trouvées par chromatographie. Il s'avère en fait que, d'après les comparaisons réalisées dans cette expérience, les valeurs trouvées à l'aide du TNBS sont nettement plus faibles (de 8 à $24 \%$ environ) que celles trouvées à l'aide de la chromatographie. L'écart moyen passe de $14,3 \%$ pour le sang portal à $17,4 \%$ pour le sang artériel, le coefficient de variation de ces écarts étant toutefois très faible $(0,8 \%)$. Ces écarts sont plus élevés que ceux mesurés par Palmer et Peters (1969). Par périodes, notamment au cours de la première heure postperfusion, ces écarts s'amenuisent surtout au niveau du sang portal : il semble que plus le sang est riche en acides aminés, et plus l'écart entre les valeurs obtenues par les deux techniques a tendance à diminuer bien que les relations curvilinéaires entre cet écart et la teneur en TNBS ne soient hautement corrélées que pour le sang artériel $(r=-0,72, n=44, P<0,01)$ pourtant moins riche en aa que le sang portal $(r=-0,59, n=44, P<0,01)$. Cela signifie que dans le cas du sang artériel, plus de la moitié de la variance de cet écart est expliquée par la teneur en azote du sang. On peut aussi supposer que ces écarts sont liés aux variations de concentrations individuelles de certains acides aminés; cependant, l'examen de l'évolution postperfusion de la composition des mélanges d'acides aminés présents dans le sang porte ou artériel ne permet de déceler aucune 
relation entre les divers acides aminés et l'importance de ces écarts. Quoi qu'il en soit, les rapports entre les valeurs obtenues par les deux techniques présentent une évolution temporelle qui n'est pas parallèle dans le sang portal et le sang artériel avec les conséquences prévisibles sur les différences portoartérielles et leurs variations selon la technique utilisée. Cependant, les divergences des valeurs portoartérielles selon la technique utilisée ne paraissent pas avoir de conséquences notables quant à la détermination quantitative de l'absorption. Si l'on en juge par les données du tableau 4, les écarts entre les quantités absorbées, selon la technique employée, n'atteignent jamais le seuil de signification. Ils sont relativement plus importants pour les quantités absorbées mesurées par unité de temps, lorsque ces quantités sont faibles, mais ils sont peu élevés pour les quantités absorbées cumulées de toute la période d'observation puisqu'ils ne varient dans un sens ou l'autre que de 2 à $14 \%$.

Dans l'étude de l'absorption des acides aminés totaux, la méthode TNBS paraît ainsi et globalement, être une méthode prévisionnelle intéressante puisque les écarts qu'elle provoque sont minimes. Elle présente, par ailleurs, des avantages certains de rapidité et de coût.

Les résultats quantitatifs d'absorption sont convergents, quelle que soit la technique utilisée. Ils confirment par ailleurs des résultats partiels obtenus par la technique TNBS et déjà publiés (Rérat et al., 1985). Il est donc inutile d'en discuter la signification mais on peut rappeler que la méthode utilisée pour quantifier l'absorption, fondée sur la mesure simultanée des différences portoartérielles et du débit sanguin dans la veine porte, permet de démontrer que l'absorption de l'azote des acides aminés est beaucoup plus rapide après perfusion duodénale d'une solution essentiellement composée de petits peptides qu'après celle d'une solution d'acides aminés libres de même composition. A souligner que ce phénomène concerne la presque totalité des acides aminés, à l'exception toutefois de la méthionine pour laquelle se produit une inversion de la hiérarchie de vitesse d'absorption selon la nature du perfusat utilisé (Rérat, 1986).

Deux types de conclusions peuvent être tirés de ce travail :

- Au plan méthodologique, la technique de dosage de l'azote aminé sanguin à l'aide d'un réactif coloré, l'acide 2, 4, 6 trinitrobenzène-1-sulfonique peut être valablement utilisée pour estimer l'aminoacidémie sanguine en vue de l'étude quantitative de l'absorption des acides aminés.

- Au plan nutritionnel, l'absorption de l'azote des acides provenant de solutions de petits peptides introduits dans le duodénum du porc est plus précoce et plus rapide que celle de l'azote provenant de solutions d'acides aminés libres. Elle est accompagnée de l'absorption d'azote endogène intestinal en quantités notables.

Reçu en décembre 1986. Accepté en juillet 1987.

\section{Références}

ARSAC M., RÉRAT A., 1962. Technique de fistulation de la veine porte chez le porc. Ann. Biol. anim. Bioch. Biophys., 2, 335-344. 
BRULE G., ROGER L., FAUQUANT M., PIOT M., 1980a. Procédé de traitement d'une matière première à base de caséine contenant des phosphocaseinates de cations bivalents, produits obtenus et applications. Brevet INRA $n^{\circ}$ 80.02.280, déposé le 1/2/1980.

BRULE G., ROGER L., FAUQUANT M., PIOT M., 1980b. Procédé de traitement d'une matière à base de caséine contenant des phosphocasénates de cations monovalents ou leurs dérivés, produits obtenus et applications. Brevet INRA $n^{\circ} 80.02 .281$, déposé le 1/2/1980.

HENRY Y., RÉRAT A., 1964. Variations des taux énergétique et azoté dans l'alimentation du Porc en croissance. Observations préliminaires. Ann. Biol. anim. Bioch. Biophys., 4, 263-271.

MOORE S., SPACKMAN D. H., STEIN W. H., 1958. Chromatography of aminoacids on sulfonated polystyrene resins. An improved system. Analyt. Chem., 30, 1185-1190.

PALMER D. W., PETERS T. Jr. 1966. Simple automatic determination of amino groups in serum plasma using trinitrobenzene sulfonate, 324-327. In SKEGGS L. T. Jr., Automation in analytical chemistry, Technicon Symp. 1965, Mediad, N.-Y., 324-327.

PALMER D. W., PETERS T. Jr., 1969. Automated determination of free amino groups in serum and plasma using 2, 4, 6-trinitrobenzene sulfonate. Clin. Chem., 15, 891-901.

PION R., FAUCONNEAU G., RÉRAT A., 1963. Etude cinétique de la composition en acides aminés du sang porte chez le porc. Ann. Biol. anim. Bioch. Biophys., 3 (hs no 1), 31-44.

PRENTON M. A., LONDON D. R., 1967. The continuous in vivo monitoring of plasma aminonitrogen. $5^{\text {th }}$ Coll. on aminoacid analysis, Technicon monograph 2, Technicon, Domont (France), 70-78.

RÉRAT A., 1981. Chronologie et bilans de l'absorption des sucres réducteurs et de l'azote $\alpha$-aminé chez le porc selon la nature des aliments. Bull. Acad. Nat. Méd., 165, 1131-1137.

RÉRAT A., 1982. Absorption des sucres et des acides aminés chez le porc, 63-87. In LAPLACE J. P., CORRING T., RÉRAT A., Digestion physiology in the pig. Coll. INRA $n^{\circ} 12$, INRAPub. Ed.

RÉRAT A., 1986. Utilisation de l'azote des aliments produits par les biotechnologies : cinétique d'absorption, métabolisation et sécrétion d'hormones pancréatiques après perfusion duodénale d'hydrolysats ménagés de protéines laitières chez le porc éveillé. Proc. Symp. int. Biotechnologie et Alimentation, Québec (sous presse).

RÉRAT A., LACROIX M., SIMOES NUNES C., VAUGELADE P., VAISSADE P., 1984. Absorption intestinale comparée d'un mélange d'hydrolysats ménagés de protéines laitières et d'un mélange d'acides aminés libres de même composition. Bull. Acad. Nat. Méd., 168, 385-391.

RÉRAT A., SIMOES NUNES C., LACROIX M., VAUGELADE P., VAISSADE P., 1985. Cinétique comparée d'apparition dans la veine porte de l'azote $\alpha$-aminé provenant de mélanges de petits peptides ou d'acides aminés libres de même composition introduits dans le duodénum chez le porc éveillé. C. R. Acad. Sci., Paris, 300, sér. III, 293-296.

RÉRAT A., VAISSADE P., VAUGELADE P., ROBIN D., ROBIN P., JUNG J., 1977. Determination of nature and quantity of endogenous nitrogen absorbed during the digestion of a proteinfree meal in the pig. Int. Symp. on aminoacids, Budapest D3, 1-8.

RÉRAT A., VAUGELADE P., 1983. Débitmétrie chronique de la veine porte chez le porc. Sci. Tech. Anim. Lab., 8, 239-248.

RÉRAT A., VAUGELADE P., VILLIERS P., 1980. A new method for measuring the absorption of nutrients in the pig : critical examination. In LOW A. G., PARTRIDGE I. G. Current concepts of digestion and absorption in pigs, NIRD, HRI, Ayr, Techn. Bull., 3, 177-216.

ROGER L., MAUBOIS J. L., BRULE G., PIOT M., 1979. Hydrolysat enzymatique total des protéines de lactosérum, obtention et application. Brevet INRA $\mathrm{N}^{\circ} 79.16 .483$, déposé le 26/6/1979.

SATAKE K., OKUYAMA T., MOCHIHIKO O., SHINODA T., 1960. The spectrophotometric determination of amine, aminoacid and peptide with 2, 4, 6-trinitrobenzene-1-sulfonic acid. $J$. Biochemistry, 47, 654-660.

WARING J. J., BOLTON W., 1967. 2, 4, 6-trinitrobenzene sulfonic acid as a colour reagent for aminoacid analysis. In $5^{\text {th }}$ Coll. on aminoacid analysis, Technicon monograph 2, Technicon Domont (France), 30-34. 